\title{
An Extension Theorem for a Sequence of Krein Space Contractions
}

\author{
Gerald Wanjala \\ Department of Mathematics and Statistics, Sultan Qaboos University, P.O. Box 36, Al-Khod, 123 Muscat, Oman \\ Correspondence should be addressed to Gerald Wanjala; wanjalag@yahoo.com
}

Received 8 December 2017; Revised 23 January 2018; Accepted 30 January 2018; Published 1 March 2018

Academic Editor: Gelu Popescu

Copyright (c) 2018 Gerald Wanjala. This is an open access article distributed under the Creative Commons Attribution License, which permits unrestricted use, distribution, and reproduction in any medium, provided the original work is properly cited.

Consider Krein spaces $\mathscr{U}$ and $\mathscr{Y}$ and let $\mathscr{H}_{k}$ and $\mathscr{K}_{k}$ be regular subspaces of $\mathscr{U}$ and $\mathscr{Y}$, respectively, such that $\mathscr{H}_{k} \subset \mathscr{H}_{k+1}$ and $\mathscr{K}_{k} \subset \mathscr{K}_{k+1}(k \in \mathbb{N})$. For each $k \in \mathbb{N}$, let $A_{k}: \mathscr{H}_{k} \rightarrow \mathscr{K}_{k}$ be a contraction. We derive necessary and sufficient conditions for the existence of a contraction $B: \mathscr{U} \rightarrow \mathscr{Y}$ such that $\left.B\right|_{\mathscr{H}_{k}}=A_{k}$. Some interesting results are proved along the way.

\section{Introduction}

A number of extensions and generalizations of classical function theoretic interpolation problems are driven by factors such as applications in systems and control theory. Many such extensions and generalizations make use of the commutant lifting theorem in one way or another. In particular, the commutant lifting theorem in the Hilbert space case which was obtained by Sz.-Nagy and Foias has been used to solve extension problems like the ones of Nevanlinna-Pick, Nudelman, Nehari, and many others. Extensions of this theorem to an indefinite setting are given in [1-5]. In [6] (see also $[7,8]$ ), a time-variant version of the commutant lifting theorem is developed. This time-variant version is called the three-chain completion theorem and is used to solve a number of nonstationary norm constrained interpolation problems on Hilbert spaces. Recall (see [6]) that the given data for the three-chain completion problem are bounded linear operators

$$
A_{k}: \mathscr{H}_{k} \longrightarrow \mathscr{K}_{k} \ominus \mathscr{M}_{k} \quad(k \in \mathbb{Z})
$$

where $\mathscr{H}_{k} \subset \mathscr{U}$ and $\mathscr{M}_{k} \subset \mathscr{K}_{k} \subset \mathcal{Y}$ for $k \in \mathbb{Z}$ are Hilbert spaces satisfying the inclusion relations

$$
\begin{aligned}
& \mathscr{H}_{k-1} \subset \mathscr{H}_{k}, \\
& \mathscr{M}_{k-1} \subset \mathscr{M}_{k},
\end{aligned}
$$

$$
\mathscr{K}_{k-1} \subset \mathscr{K}_{k}
$$$$
(k \in \mathbb{Z}) .
$$

Given operators (1) and tolerance $\gamma$ the problem is to find an operator $B: \mathscr{U} \rightarrow \mathscr{Y}$ such that $\|B\| \leq \gamma$ and

$$
\begin{array}{r}
B \mathscr{H}_{k} \subset \mathscr{K}_{k}, \\
\left.\left(I-P_{\mathscr{M}_{k}}\right) B\right|_{\mathscr{H}_{k}}=A_{k}
\end{array}
$$

$$
(k \in \mathbb{Z}) .
$$

As far as we know, no extension of this theorem to an indefinite setting has been developed so far.

The three-chain theorem mentioned above is the motivation for the extension problem considered in this paper. For $k \in \mathbb{N}$, consider a sequence of Krein space contractions $A_{k}: \mathscr{H}_{k} \rightarrow \mathscr{K}_{k}$, where $\mathscr{H}_{k}$ and $\mathscr{K}_{k}$ are nested regular subspaces of some Krein spaces $\mathcal{U}$ and $\mathscr{Y}$, respectively. The problem is to find a contraction $B: \mathscr{U} \rightarrow \mathscr{Y}$ such that

$$
B h=A_{k} h
$$

for all $h \in \mathscr{H}_{k}$.

In order to keep this paper as self-contained as possible, we briefly outline some definitions and some elementary facts about Krein spaces and bounded linear operators defined 
on them. This is done in Section 2. Some important results are stated and proved in Section 3. An extension theorem is considered in Section 4 while Section 5 contains some simple application of the extension theorem discussed in Section 4.

\section{Preliminaries}

In this section we recall some definitions and basic notions of indefinite metric space theory. More details can be obtained from $[2,9-12]$.

Let $\mathscr{K}$ be a linear space and let $\langle\cdot, \cdot\rangle$ be a sesquilinear form on $\mathscr{K}$. This sesquilinear form is called an indefinite metric on $\mathscr{K}$. If $\mathscr{K}$ admits a direct orthogonal sum decomposition

$$
\mathscr{K}=\mathscr{K}^{+} \oplus \mathscr{K}^{-}
$$

in which $\left(\mathscr{K}^{ \pm}, \pm\langle\cdot, \cdot\rangle\right)$ are Hilbert spaces, then $\mathscr{K}$ (or $(\mathscr{K},\langle\cdot, \cdot\rangle))$ is called a Krein space. Decomposition (5), which is not unique in general, is called a fundamental decomposition and gives rise to orthogonal projections from $\mathscr{K}$ onto $\mathscr{K}^{ \pm}$, which we denote by $P^{ \pm}$, respectively. The self-adjoint and unitary operator $J$ on $\mathscr{K}$ defined by $J=P^{+}-P^{-}$, is called a fundamental symmetry on $\mathscr{K}$.

The space $\mathscr{K}$ with the inner product

$$
\begin{aligned}
{[x, y]=\langle J x, y\rangle=\left\langle x_{+}, y_{+}\right\rangle-\left\langle x_{-}, y_{-}\right\rangle, } & \\
& x_{ \pm}, y_{ \pm} \in \mathscr{K}^{ \pm}
\end{aligned}
$$

is a Hilbert space. This inner product is used to define the norm of an element $x$ of the Krein space $\mathscr{K}$ by

$$
\|x\|^{2}=[x, x] .
$$

Topological notions such as convergence and continuity are understood to be with respect to this norm topology. The inner product $[\cdot, \cdot]$ in (6) depends on decomposition (5), as does the norm $\|\cdot\|$ in (7), but the norms generated by different decompositions of $\mathscr{K}$ are equivalent. We will denote the norm $\|\cdot\|$ in (7) by $\|\cdot\|_{J}$ where clarity is needed.

An orthogonal projection in a Krein space is a bounded self-adjoint operator $R$ in $\mathscr{K}$ such that $R^{2}=R$. Note that the norm of an orthogonal projection in a Krein space need not be less than 1 . The range $\mathscr{M}$ of an orthogonal projection $R$ in a Krein space $\mathscr{K}$ is a closed subspace of $\mathscr{K}$ and the space $\mathscr{K}$ can be decomposed as

$$
\mathscr{K}=\mathscr{M} \oplus \mathscr{M}^{\perp}
$$

where $\mathscr{M}^{\perp}=\{l \in \mathscr{K}:\langle l, m\rangle=0 \forall m \in \mathscr{M}\}$. On the other hand, given a closed subspace $\mathscr{M}$ of $\mathscr{K}$ such that decomposition (8) holds, then $\mathscr{M}$ is the range of an orthogonal projection in $\mathscr{K}$. In this case, $(\mathscr{M},\langle\cdot, \cdot\rangle)$ is again a Krein space. Unlike in the Hilbert space case, the decomposition (8) need not hold for a given closed subspace. Closed subspaces for which this decomposition holds are referred to as regular subspaces.

Let $\mathscr{H}$ and $\mathscr{K}$ be Krein spaces and let $T: \mathscr{H} \rightarrow \mathscr{K}$ be a bounded linear operator. We say that $T$ is a contraction if for all $x \in \mathscr{H},\langle T x, T x\rangle_{\mathscr{K}} \leq\langle x, x\rangle_{\mathscr{H}}$. If both $T$ and its Krein space adjoint $T^{*}$ are contractions, then $T$ is called a bicontraction.
Let $\mathscr{H}$ and $\mathscr{K}$ be Krein spaces and let $T \in B(\mathscr{H}, \mathscr{K})$, the space of bounded linear operators from $\mathscr{H}$ into $\mathscr{K}$. A column extension of $T$ is an operator of the form

$$
C=\left(\begin{array}{l}
T \\
E
\end{array}\right): \mathscr{H} \longrightarrow\left(\begin{array}{l}
\mathscr{K} \\
\mathscr{E}
\end{array}\right),
$$

where $\mathscr{E}$ is a Krein space and $E \in B(\mathscr{H}, \mathscr{E})$. By a row extension of $T$ we shall mean an operator of the form

$$
L=\left(\begin{array}{ll}
T & E
\end{array}\right):\left(\begin{array}{l}
\mathscr{H} \\
\mathscr{E}
\end{array}\right) \longrightarrow \mathscr{K},
$$

where $\mathscr{E}$ is a Krein space and $E \in B(\mathscr{E}, \mathscr{K})$. It is shown in [2] (see also [3]) that if $T$ is a contraction, then there exist contractive row and column extensions of $T$ where the extension space is a Hilbert space. Contractive $2 \times 2$ matrix extensions of a contractive operator $T: \mathscr{H} \rightarrow \mathscr{K}$ where the extending space $\mathscr{E}$ is a Hilbert space are thoroughly discussed in [2] where Lemma 1, Theorem 2, and Lemma 3 can be found. Results more general than those provided by Lemma 1 and Theorem 2 can be found in [1] while Lemma 3 can also be found in [4].

Lemma 1. Let $\mathscr{H}$ and $\mathscr{K}$ be Krein spaces and let $T \in B(\mathscr{H}, \mathscr{K})$ be a contraction. Let

$$
C=\left(\begin{array}{c}
T \\
\widetilde{E}^{*}
\end{array}\right) \in B(\mathscr{H}, \mathscr{K} \oplus \widetilde{\mathscr{E}})
$$

be a contractive column extension of $T$ with $\widetilde{\mathscr{E}}$ a Hilbert space. If norms are computed relative to some fixed fundamental decompositions of $\mathscr{H}$ and $\mathscr{K}$ and the induced fundamental decomposition of $\mathscr{K} \oplus \widetilde{\mathscr{E}}$, then

$$
\|C\|^{2} \leq 1+2\|T\|^{2} \text {. }
$$

The above norm estimate enables one to fix a bound for the norm of the operator $C$. The following lemma is helpful in finding $2 \times 2$ matrix extensions of a contractive operator $T$. See [13] for a similar result in a Hilbert space setting.

Theorem 2. Let $\mathscr{H}, \mathscr{K}$, and $\mathscr{E}$ be Krein spaces and let $\widetilde{\mathscr{E}}$ be a Hilbert space. Assume that

$$
\begin{array}{r}
\left(\begin{array}{ll}
C_{11} & C_{12}
\end{array}\right):\left(\begin{array}{l}
\mathscr{H} \\
\mathscr{E}
\end{array}\right) \longrightarrow \mathscr{K}, \\
\left(\begin{array}{l}
C_{11} \\
C_{21}
\end{array}\right): \mathscr{H} \longrightarrow\left(\begin{array}{c}
\mathscr{K} \\
\widetilde{\mathscr{E}}
\end{array}\right)
\end{array}
$$

are contractions. Then there exists an operator $C_{22}: \mathscr{E} \rightarrow \widetilde{\mathscr{E}}$ such that

$$
C=\left(\begin{array}{ll}
C_{11} & C_{12} \\
C_{21} & C_{22}
\end{array}\right):\left(\begin{array}{l}
\mathscr{H} \\
\mathscr{E}
\end{array}\right) \longrightarrow\left(\begin{array}{c}
\mathscr{K} \\
\widetilde{\mathscr{E}}
\end{array}\right)
$$

is a contraction. If

$$
\left(\begin{array}{ll}
C_{11} & C_{12}
\end{array}\right)
$$

is a bicontraction, $C_{22}$ may be chosen such that $C$ is a bicontraction. 
We conclude this section by stating the following lemma.

Lemma 3. Let $\mathcal{N}_{1}, \mathcal{N}_{2}, \ldots$ be regular subspaces of a Krein space $\mathscr{H}$ such that $\mathcal{N}_{1} \supset \mathcal{N}_{2} \supset \cdots$. Suppose that the projections $P_{1}, P_{2}, \ldots$ of $\mathscr{H}$ onto the subspaces $\mathcal{N}_{1}, \mathcal{N}_{2}, \ldots$ are uniformly bounded. Then

$$
\begin{aligned}
& \mathcal{N}=\bigcap_{n=1}^{\infty} \mathcal{N}_{n}, \\
& \mathscr{M}=\overline{\operatorname{span}} \mathcal{N}_{n}^{\perp}, \\
& n=1,2, \ldots
\end{aligned}
$$

are regular subspaces of $\mathscr{H}$ with $\mathscr{N}=\mathscr{M}^{\perp}$. If $P$ is the projection of $\mathscr{H}$ onto $\mathcal{N}$, then $P=\lim _{n \rightarrow \infty} P_{n}$ with convergence in the strong operator topology.

\section{Some Preliminary Results}

In this section we prove some useful results regarding sequences of regular subspaces. See [4, Corollary 3.3 and Lemma 3.4] for closely related results.

Theorem 4. For $n \geq 1$, let $\mathscr{L}_{n}$ be a sequence of regular subspaces of a Krein space $\mathscr{H}$ such that $\mathscr{L}_{n} \subset \mathscr{L}_{n+1}, n \in \mathbb{N}$. For each $n \in \mathbb{N}$, let $P_{n}$ be the projection of $\mathscr{H}$ onto $\mathscr{L}_{n}$. If the projections $P_{n}$ are uniformly bounded then $\mathscr{L}=\overline{\operatorname{span}} \mathscr{L}_{n}$ is a regular subspace of $\mathscr{H}$.

Proof. Set $\mathscr{N}_{1}=\mathscr{L}_{1}^{\perp}, \mathcal{N}_{2}=\mathscr{L}_{2}^{\perp}, \ldots$ Then $\mathscr{N}_{1}, \mathcal{N}_{2}, \ldots$ is a sequence of regular subspaces of $\mathscr{H}$ such that $\mathscr{N}_{1} \supset \mathscr{N}_{2} \supset$ $\cdots$. Let $Q_{1}, Q_{2}, \ldots$ be the projections of $\mathscr{H}$ onto the subspaces $\mathcal{N}_{1}, \mathcal{N}_{2}, \ldots$. Then $Q_{n}=\left(1-P_{n}\right)$ and so $\left\|Q_{n}\right\| \leq 1+\left\|P_{n}\right\|$. Since the projections $P_{n}$ are uniformly bounded we see that the projections $Q_{n}$ are also uniformly bounded. Hence $\mathscr{L}$ is a regular subspace of $\mathscr{H}$ by Lemma 3.

Theorem 5. For $n \geq 1$, let $\mathscr{L}_{n}$ be regular subspaces of a Krein space $\mathscr{K}$ such that $\mathscr{L}_{n} \subset \mathscr{L}_{n+1}$. Let $P_{n}$ be the projection of $\mathscr{K}$ onto $\mathscr{L}_{n}$. If $\mathscr{L}_{n}=\mathscr{L}_{n}^{+} \oplus \mathscr{L}_{n}^{-}$is a fundamental decomposition of $\mathscr{L}_{n}$, we denote by $Q_{n}^{ \pm}$the projection of $\mathscr{L}_{n}$ onto $\mathscr{L}_{n}^{ \pm}$. Let

$$
\mathscr{L}=\overline{\operatorname{span}} \mathscr{L}_{n} .
$$

Then the following are equivalent:

(1) $\mathscr{L}$ is regular and there exists a fundamental symmetry $J$ on $\mathscr{L}$ such that $J \mid \mathscr{L}_{n}$ is a fundamental symmetry on $\mathscr{L}_{n}$.

(2) There exist fundamental decompositions $\mathscr{L}_{n}=\mathscr{L}_{n}^{+} \oplus$ $\mathscr{L}_{n}^{-}$such that

$$
\begin{aligned}
& \text { (a) } \mathscr{L}_{n}^{ \pm} \subset \mathscr{L}_{n+1}^{ \pm} \text {, } \\
& \text { (b) } \sup \left\|Q_{n}^{ \pm} P_{n}\right\|_{\widetilde{J}}<\infty \text {, }
\end{aligned}
$$

where $\widetilde{J}$ is any fundamental symmetry on $\mathscr{K}$.

Proof. Suppose that (1) holds and let $\mathscr{L}=\mathscr{L}_{+} \oplus \mathscr{L}_{-}$be the fundamental decomposition of $\mathscr{L}$ which gives rise to the fundamental symmetry $J=Q_{+}-Q_{-}$with the stated property, where $Q_{ \pm} \mathscr{L}=\mathscr{L}_{ \pm}$. Then $J \mid \mathscr{L}_{n}=Q_{n}^{+}-Q_{n}^{-}$where $Q_{n}^{ \pm} \mathscr{L}_{n}=$ $\mathscr{L}_{n}^{ \pm}$. To show that (a) holds, we let $x \in \mathscr{L}_{n}^{+}$. Then

$$
x=Q_{n}^{+} x-Q_{n}^{-} x=J x=Q_{n+1}^{+} x-Q_{n+1}^{-} x .
$$

Hence $x=Q_{n+1}^{+} x-Q_{n+1}^{-} x$ and so $\left(I-Q_{n+1}^{+}\right) x=-Q_{n+1}^{-} x$. This means that $Q_{n+1}^{-} x=-Q_{n+1}^{-} x$ and that $Q_{n+1}^{-} x=0$. Therefore $x=Q_{n+1}^{+} x \in \mathscr{L}_{n+1}^{+}$. Similarly, we have that $\mathscr{L}_{n}^{-} \subset \mathscr{L}_{n+1}^{-}$. Hence (a) holds. To show that (b) also holds, we let

$$
y \in \mathscr{K}=\mathscr{L}_{n} \oplus\left(\mathscr{L} \ominus \mathscr{L}_{n}\right) \oplus \mathscr{L}^{\perp} .
$$

Let $J_{n}=J\left|\mathscr{L}_{n}, \widehat{J}_{n}=J\right|\left(\mathscr{L} \ominus \mathscr{L}_{n}\right)$ and let $J_{\perp}$ be a fundamental symmetry on $\mathscr{L}^{\perp}$. Then $\widetilde{J}:=J+J_{\perp}$ is a fundamental symmetry on $\mathscr{K}$. Now,

$$
\begin{aligned}
\|y\|_{\mathscr{K}, \tilde{J}}^{2} & =\langle\widetilde{J} y, y\rangle \\
& =\left\langle\widetilde{J}\left(x_{n}+y_{n}+z_{n}\right),\left(x_{n}+y_{n}+z_{n}\right)\right\rangle \\
& =\left\langle J_{n} x_{n}, x_{n}\right\rangle+\left\langle\widehat{J}_{n} y_{n}, y_{n}\right\rangle+\left\langle J_{\perp} z_{n}, z_{n}\right\rangle,
\end{aligned}
$$

where $y=x_{n}+y_{n}+z_{n}$ with $x_{n} \in \mathscr{L}_{n}, y_{n} \in\left(\mathscr{L} \ominus \mathscr{L}_{n}\right)$ and $z_{n} \in \mathscr{L}^{\perp}$. Therefore

$$
\begin{aligned}
\left\|Q_{n}^{+} P_{n} y\right\|_{\mathscr{L}_{n}, J_{n}}^{2} & =\left\|Q_{n}^{+} x_{n}\right\|_{\mathscr{L}_{n}, J_{n}}^{2}=\left\langle J_{n} Q_{n}^{+} x_{n}, Q_{n}^{+} x_{n}\right\rangle \\
& =\left\langle Q_{n}^{+} x_{n}, Q_{n}^{+} x_{n}\right\rangle=\left\langle Q_{n}^{+} x_{n}, x_{n}\right\rangle \\
& =\left\langle\left(Q_{n}^{+}-Q_{n}^{-}\right) x_{n}, x_{n}\right\rangle+\left\langle Q_{n}^{-} x_{n}, x_{n}\right\rangle \\
& \leq\left\langle\left(Q_{n}^{+}-Q_{n}^{-}\right) x_{n}, x_{n}\right\rangle=\left\langle J_{n} x_{n}, x_{n}\right\rangle \\
& \leq\left\langle J_{n} x_{n}, x_{n}\right\rangle+\left\langle\widehat{J}_{n} y_{n}, y_{n}\right\rangle+\left\langle J_{\perp} z_{n}, z_{n}\right\rangle \\
& =\|y\|_{\mathscr{K}}^{2} .
\end{aligned}
$$

Hence $\left\|Q_{n}^{+} P_{n}\right\| \leq 1$. Similarly $\left\|Q_{n}^{-} P_{n}\right\| \leq 1$.

Conversely, assume that (2) holds. We start by showing that $\mathscr{L}$ is regular. To do this we note that $P_{n}=\left(Q_{n}^{+}+Q_{n}^{-}\right) P_{n}$. So $\left\|P_{n}\right\| \leq\left\|Q_{n}^{+} P_{n}\right\|+\left\|Q_{n}^{-} P_{n}\right\| \leq M_{1}$ for some constant $M_{1}>0$. Hence $\mathscr{L}=\overline{\operatorname{span}} \mathscr{L}_{n}$ is regular by Theorem 4 .

Next set $\mathscr{N}_{n}=\left(\mathscr{L}_{n}^{+}\right)^{\perp}$ and let $R_{n}$ be the projection of $\mathscr{K}$ onto $\mathscr{N}_{n}=\left(\mathscr{L}_{n}^{+}\right)^{\perp}$. Then $R_{n}=I-Q_{n}^{+} P_{n}$. Hence $\left\|R_{n}\right\| \leq 1+\left\|Q_{n}^{+} P_{n}\right\| \leq M_{2}$ for some constant $M_{2}>0$ since (2) holds. Hence $\mathcal{N}^{+}:=\bigcap \mathscr{N}_{n}$ and $\mathscr{M}^{+}:=\frac{2}{\operatorname{span}} \mathscr{L}_{n}^{+}$ are regular subspaces by Lemma 3 . Let $x \in \mathscr{M}^{+}$. Then there exists a sequence $x_{n} \in \operatorname{span} \mathscr{L}_{n}^{+}$such that $x_{n} \rightarrow x$. Hence $\left\langle x_{n}, x_{n}\right\rangle \rightarrow\langle x, x\rangle$. This means that $\langle x, x\rangle \geq 0$ since $\left\langle x_{n}, x_{n}\right\rangle \geq 0$. Hence $\mathscr{M}^{+}$is regular and nonnegative and so it is a uniformly positive subspace of $\mathscr{L}$. Similarly $\mathscr{M}^{-}:=$ $\overline{\operatorname{span}} \mathscr{L}_{n}^{-}$is a uniformly negative subspace of $\mathscr{L}$. We now show that

$$
\mathscr{L}=\mathscr{M}^{+} \oplus \mathscr{M}^{-}
$$


Let $x \in \mathscr{M}^{+}$. Then there exists a sequence $x_{n} \in \operatorname{span} \mathscr{L}_{n}^{+}$ such that $x_{n} \rightarrow x$. If $y \in \mathscr{M}^{-}$then there exists a sequence $y_{n} \in \operatorname{span} \mathscr{L}_{n}^{-}$such that $y_{n} \rightarrow y$. Hence $\langle x, y\rangle=\lim \left\langle x_{n}, y_{n}\right\rangle$ $=0$. This implies that $\mathscr{M}^{+} \perp \mathscr{M}^{-}$. Next, assume that $u \in$ $\mathscr{L} \ominus\left(\mathscr{M}^{+} \oplus \mathscr{M}^{-}\right)$. Then $u \perp \mathscr{M}^{+}$and $u \perp \mathscr{M}^{-}$. This in turn means that $u \perp \mathscr{L}_{n}^{+}$and $u \perp \mathscr{L}_{n}^{-}$and so $u \perp \mathscr{L}_{n}$. Let $v \in \mathscr{L}$. Then there exists a sequence $v_{n} \in \operatorname{span} \mathscr{L}_{n}$ such that $v_{n} \rightarrow v$. Hence $\langle u, v\rangle=\lim \left\langle u, v_{n}\right\rangle=0$ for all $v \in \mathscr{L}$ and so $v=0$. Hence $\mathscr{L}=\mathscr{M}^{+} \oplus \mathscr{M}^{-}$. This implies that $\mathscr{M}^{+}$is maximal positive and $\mathscr{M}^{-}$is maximal negative. Hence $\mathscr{M}^{+}$is a maximal uniformly positive space and $\mathscr{M}^{-}$is a maximal uniformly negative space. Since $\mathscr{M}^{+} \perp \mathscr{M}^{-}, \mathscr{M}^{+}$is uniformly positive and $\mathscr{M}^{-}$is uniformly negative, there exists a fundamental decomposition $\mathscr{L}=\mathscr{L}_{+} \oplus \mathscr{L}_{-}$such that $\mathscr{M}^{ \pm} \subset \mathscr{L}_{ \pm}$. But $\mathscr{M}^{ \pm}$maximal implies $\mathscr{M}^{ \pm}=\mathscr{L}_{ \pm}$. Hence $\mathscr{L}=\mathscr{M}^{+} \oplus \mathscr{M}^{-}$ is a fundamental decomposition of $\mathscr{L}$. This decomposition gives rise to a fundamental symmetry $J=Q_{+}-Q_{-}$where $Q_{ \pm} \mathscr{L}=\mathscr{M}^{ \pm}$.

We show that $J \mid \mathscr{L}_{n}=Q_{n}^{+}-Q_{n}^{-}$. Let $x \in \mathscr{L}_{n} \subset \mathscr{L}$. Then $x=x_{+}+x_{-}, x_{ \pm} \in \mathscr{L}_{n}^{ \pm} \subset \frac{n}{\operatorname{span}} \mathscr{L}_{n}^{ \pm}=\mathscr{M}^{ \pm}$. Hence $Q_{+} x=Q_{+}\left(x_{+}+x_{-}\right)=x_{+}=Q_{n}^{+} x$. Similarly, $Q_{-} x=Q_{n}^{-} x$ and so $J \mid \mathscr{L}_{n}=Q_{n}^{+}-Q_{n}^{-}$.

Corollary 6. For $n \geq 1$, let $\mathscr{K}, \mathscr{L}_{n}, \mathscr{L}$, and $P_{n}$ be as in Theorem 5. Then there exists a fundamental symmetry $J$ on $\mathscr{K}$ which commutes with the projections $P_{n}$ onto $\mathscr{L}_{n}$ if and only if $\mathscr{L}$ is regular and there exists a fundamental symmetry $J^{\prime}$ on $\mathscr{L}$ such that $J^{\prime} \mid \mathscr{L}_{n}$ is a fundamental symmetry on $\mathscr{L}_{n}$.

Proof. Suppose that $\mathscr{L}$ is regular and $J^{\prime}$ exists which has the stated property. Let $\mathscr{L}=\mathscr{L}_{+} \oplus \mathscr{L}_{-}$be the fundamental decomposition which gives rise to $J^{\prime}$. Since $\mathscr{L}$ is regular there exists a fundamental decomposition $\mathscr{K}=\mathscr{K}_{+} \oplus \mathscr{K}_{-}$such that $\mathscr{L}_{ \pm} \subset \mathscr{K}_{ \pm}$. This gives rise to $J$ on $\mathscr{K}$ such that $J \mid \mathscr{L}_{n}$ is a fundamental symmetry on $\mathscr{L}_{n}$. Let $f \in \mathscr{K}$. Then $f=f_{n}+f_{n}^{\perp}$, where $f_{n} \in \mathscr{L}_{n}$ and $f_{n}^{\perp} \in \mathscr{L}_{n}^{\perp}$. Now, $J P_{n} f=J f_{n}=f_{n}^{+}-f_{n}^{-}$ (where $f_{n}^{ \pm} \in \mathscr{L}_{n}^{ \pm}, \mathscr{L}_{n}=\mathscr{L}_{n}^{+} \oplus \mathscr{L}_{n}^{-}$). On the other hand,

$$
\begin{aligned}
P_{n} J f & =P_{n} J\left(f_{n}+f_{n}^{\perp}\right) \\
& =P_{n} J\left[\left(f_{n}^{+}+f_{n}^{-}\right)+\left(f_{n}^{\perp+}+f_{n}^{\perp-}\right)\right] \\
& =P_{n}\left[J\left(f_{n}^{+}+f_{n}^{-}\right)+J\left(f_{n}^{\perp+}+f_{n}^{\perp-}\right)\right] \\
& =P_{n}\left(f_{n}^{+}-f_{n}^{-}\right)+P_{n}\left(f_{n}^{\perp+}-f_{n}^{\perp-}\right)=f_{n}^{+}-f_{n}^{-} .
\end{aligned}
$$

Hence $J P_{n}=P_{n} J$.

Conversely, assume that there exists a fundamental symmetry $J$ on $\mathscr{K}$ that commutes with the projections $P_{n}$. To show that $P_{n}$ 's are uniformly bounded we consider the fundamental decomposition $\mathscr{K}=\mathscr{K}_{+} \oplus \mathscr{K}_{-}$which gives rise to the fundamental symmetry $J$. Let

$$
P_{n}=\left(\begin{array}{cc}
A_{11}^{n} & A_{12}^{n} \\
A_{21}^{n} & A_{22}^{n}
\end{array}\right):\left(\begin{array}{c}
\mathscr{K}_{+} \\
\mathscr{K}_{-}
\end{array}\right) \longrightarrow\left(\begin{array}{c}
\mathscr{L}_{n}^{+} \\
\mathscr{L}_{n}^{-}
\end{array}\right)
$$

be the matrix representation of $P_{n}$ with respect to these decompositions. Then the commutativity condition

$$
\left(\begin{array}{cc}
1 & 0 \\
0 & -1
\end{array}\right)\left(\begin{array}{cc}
A_{11}^{n} & A_{12}^{n} \\
A_{21}^{n} & A_{22}^{n}
\end{array}\right)=\left(\begin{array}{cc}
A_{11}^{n} & A_{12}^{n} \\
A_{21}^{n} & A_{22}^{n}
\end{array}\right)\left(\begin{array}{cc}
1 & 0 \\
0 & -1
\end{array}\right)
$$

implies that $A_{21}^{n}=A_{12}^{n}=0$ and so the matrix representation of $P_{n}$ is diagonal; that is to say,

$$
P_{n}=\left(\begin{array}{cc}
A_{11}^{n} & 0 \\
0 & A_{22}^{n}
\end{array}\right):\left(\begin{array}{c}
\mathscr{K}_{+} \\
\mathscr{K}_{-}
\end{array}\right) \rightarrow\left(\begin{array}{c}
\mathscr{L}_{n}^{+} \\
\mathscr{L}_{n}^{-}
\end{array}\right) .
$$

Since $\left\|A_{11}^{n}\right\| \leq 1$ and $\left\|A_{22}^{n}\right\| \leq 1$ for all $n \in \mathbb{N}$, we see that $P_{n}$ 's are uniformly bounded. The uniform boundedness of $P_{n}$ 's implies that $\mathscr{L}$ is regular. From the matrix representation of $P_{n}$ above we see that $A_{11}^{n}=Q_{n}^{+} P_{n}$ and $A_{22}^{n}=Q_{n}^{-} P_{n}$ and so $\sup \left\|Q_{n}^{ \pm} P_{n}\right\|<\infty$. Hence condition (b) in Theorem 5 holds. Since, for each $n \in \mathbb{N}, \mathscr{L}_{n}$ is a regular subspace and $\mathscr{L}_{n} \subset$ $\mathscr{L}_{n+1}$, we have that, for any fundamental decomposition $\mathscr{L}_{n}^{n+1} \mathscr{L}_{n}^{+} \oplus \mathscr{L}_{n}^{-}$, there exist a fundamental decomposition

$$
\mathscr{L}_{n+1}=\mathscr{L}_{n+1}^{+} \oplus \mathscr{L}_{n+1}^{-}
$$

such that $\mathscr{L}_{n}^{ \pm} \subset \mathscr{L}_{n+1}^{ \pm}$. Hence condition (a) in Theorem 5 also holds. This shows that part (1) of Theorem 5 holds and this completes the proof.

\section{An Extension Theorem for a Sequence of Contractions}

In this section, we formulate and give a proof of an extension theorem for a sequence of contractions defined on a nested sequence of regular subspaces. Please refer to [14, Lemma 3.1] and [5] for closely related results.

Theorem 7. Let $\mathcal{U}$ and $\mathscr{Y}$ be Krein spaces and let $\mathscr{H}_{k} \subset \mathcal{U}$ and $\mathscr{K}_{k} \subset \mathcal{Y}, k \in \mathbb{N}$, be sequences of regular subspaces satisfying

$$
\begin{aligned}
& \mathscr{H}_{k} \subset \mathscr{H}_{k+1}, \\
& \mathscr{K}_{k} \subset \mathscr{K}_{k+1} .
\end{aligned}
$$

Let $P_{\mathscr{H}_{k}}$ and $P_{\mathscr{K}_{k}}$ be the orthogonal projections of $\mathscr{U}$ onto $\mathscr{H}_{k}$ and $\mathscr{Y}$ onto $\mathscr{K}_{k}$, respectively, and let $\widetilde{\mathscr{H}}=\overline{\operatorname{span}} \mathscr{H}_{k}$ and $\widetilde{\mathscr{K}}=$ $\overline{\operatorname{span}} \mathscr{K}_{k}$. Assume that there exist fundamental symmetries $J$ on $\mathcal{U}$ and $J^{\prime}$ on $\mathcal{Y}$ such that $J$ commutes with $P_{\mathscr{H}_{k}}$ and $J^{\prime}$ commutes with $P_{\mathscr{K}_{k}}$ and that $\mathscr{U} \ominus \widetilde{\mathscr{H}}, \mathcal{Y} \ominus \widetilde{\mathscr{K}}, \mathscr{H}_{k+1} \ominus \mathscr{H}_{1}$, and $\mathscr{K}_{k+1} \ominus \mathscr{K}_{1}$ are all Hilbert space.

For each $k \in \mathbb{N}$, let

$$
A_{k}: \mathscr{H}_{k} \longrightarrow \mathscr{K}_{k}
$$

be a contraction. Then there exists a contraction $B: \mathcal{U} \rightarrow \mathcal{Y}$ such that

$$
\left.B\right|_{\mathscr{H}_{k}}=A_{k}, \quad k \in \mathbb{N}
$$

if and only if

$$
\begin{gathered}
\left.A_{k+1}\right|_{\mathscr{H}_{k}}=A_{k}, \\
\sup _{k \in \mathbb{N}}\left\|A_{k}\right\|<\infty .
\end{gathered}
$$


Proof. First, let us assume that such an extension $B \in$ $B(\mathscr{U}, \mathcal{Y})$ exists and take $h_{k} \in \mathscr{H}_{k}$. Since $\mathscr{H}_{k} \subset \mathscr{H}_{k+1}$ and (30) holds, we have that

$$
A_{k+1} h_{k}=B h_{k}=A_{k} h_{k}
$$

and therefore $\left.A_{k+1}\right|_{\mathscr{H}_{k}}=A_{k}$. Hence the first condition in (31) holds. To show that the second condition also holds, we first note that since $J$ and $J^{\prime}$ commute with $P_{\mathscr{H}_{k}}$ and $P_{\mathscr{K}_{k}}$, respectively, Corollary 6 ensures that the subspaces $\widetilde{\mathscr{H}}$ and $\widetilde{\mathscr{K}}$ are regular and that there exist fundamental symmetries $J_{\widetilde{\mathscr{H}}}$ on $\widetilde{\mathscr{H}}$ and $J_{\widetilde{\mathscr{K}}}^{\prime}$ on $\widetilde{\mathscr{K}}$ such that $J_{\widetilde{\mathscr{H}}} \mid \mathscr{H}_{k}$ is a fundamental symmetry on $\mathscr{H}_{k}$ and $J_{\mathscr{K}}^{\prime} \mid \mathscr{K}_{k}$ is a fundamental symmetry on $\mathscr{K}_{k}$. Let $h \in \mathscr{H}_{k}$. Then we have that $A_{k} h B h$. Hence,

$$
\begin{aligned}
\left\|A_{k} h\right\|_{\widetilde{\mathscr{K}}} & =\left\|A_{k} h\right\|_{\mathscr{K}_{k}}=\left\langle J_{\mathscr{\mathscr { K }}^{\prime}}^{\prime} A_{k} h, A_{k} h\right\rangle \\
& =\left\langle J_{\widetilde{\mathscr{K}}}^{\prime} B h, B h\right\rangle=\|B h\|_{\widetilde{\mathscr{K}}}=\|B\|\|h\|_{\mathscr{\mathscr { H }}} .
\end{aligned}
$$

Hence, $\left\|A_{k}\right\| \leq\|B\|$ for all $k \in \mathbb{N}$ and so the norms of $A_{k}$ 's are uniformly bounded.

Conversely, let $A_{k}: \mathscr{H}_{k} \rightarrow \mathscr{K}_{k}, k \in \mathbb{N}$, be contractions satisfying both conditions in (31). Since $\left.A_{k+1}\right|_{\mathscr{H}_{k}}=A_{k}$, we can decompose the operator

$$
\left.A_{k+1}\right|_{\mathscr{H}_{k}}: \mathscr{H}_{k} \longrightarrow\left(\begin{array}{c}
\mathscr{K}_{k} \\
\mathscr{K}_{k+1} \ominus \mathscr{K}_{k}
\end{array}\right)
$$

as $\left.A_{k+1}\right|_{\mathscr{H}_{k}}=\left(\begin{array}{c}A_{k} \\ 0\end{array}\right)$. The fact that $A_{k}$ is a contraction and $\mathscr{H}_{k+1} \ominus \mathscr{H}_{k}$ is a Hilbert space implies that the operator

$$
\left(\begin{array}{ll}
A_{k} & 0
\end{array}\right):\left(\begin{array}{c}
\mathscr{H}_{k} \\
\mathscr{H}_{k+1} \ominus \mathscr{H}_{k}
\end{array}\right) \longrightarrow \mathscr{K}_{k}
$$

is a contraction. Since $\left.A_{k+1}\right|_{\mathscr{H}_{k}}=\left(\begin{array}{c}A_{k} \\ 0\end{array}\right)$ is a contraction, Theorem 2 implies that there exists a bounded operator $c_{22}$ : $\mathscr{H}_{k+1} \ominus \mathscr{H}_{k} \rightarrow \mathscr{K}_{k+1} \ominus \mathscr{K}_{k}$ such that

$$
C_{k+1}=\left(\begin{array}{cc}
A_{k} & 0 \\
0 & c_{22}
\end{array}\right):\left(\begin{array}{c}
\mathscr{H}_{k} \\
\mathscr{H}_{k+1} \ominus \mathscr{H}_{k}
\end{array}\right) \rightarrow\left(\begin{array}{c}
\mathscr{K}_{k} \\
\mathscr{K}_{k+1} \ominus \mathscr{K}_{k}
\end{array}\right)
$$

is a contraction. For each $k=0,1,2, \ldots$, the operator $C_{k+1}$ is clearly a contractive extension of $A_{k}$.

We now show that, for $k=0,1,2, \ldots$, the contractive liftings $C_{k+1}$ 's of $A_{k}$ 's are uniformly bounded. Let $M$ be the bound for the norm of $A_{k}$ 's. Then $\left\|\left(\begin{array}{ll}A_{k} & 0\end{array}\right)\right\|=\left\|A_{k}\right\| \leq M$ and so Lemma 1 implies that $\left\|C_{k+1}\right\|^{2} \leq 1+2\left\|A_{k}\right\|^{2} \leq 1+2 M^{2}$. Hence $C_{k+1}$ 's are uniformly bounded.

Define an operator $C_{\infty}^{\prime}: \operatorname{span} \mathscr{H}_{k} \rightarrow \operatorname{span} \mathscr{K}_{k}$ by $C_{\infty}^{\prime} x=$ $C_{k+1} x$ for $x \in \mathscr{H}_{k}$. The operator $C_{\infty}^{\prime}$ is well defined. To see this, assume that $x \in \mathscr{H}_{k}$ and $x \in \mathscr{H}_{l}$. Then $C_{\infty}^{\prime} x=C_{k+1} x$ and $C_{\infty}^{\prime} x=C_{l+1} x$. For $k<l,\left.C_{l+1}\right|_{\mathscr{H}_{k}}=\left.A_{l}\right|_{\mathscr{H}_{k}}=A_{k}=$ $\left.C_{k+1}\right|_{\mathscr{H}_{k}}$. The operator $C_{\infty}^{\prime}$ is bounded since the contractions $C_{k+1}$ are uniformly bounded. To see that this is the case, consider $x \in \mathscr{H}_{k}$. Then

$$
\begin{aligned}
{[x, x]_{\mathscr{\mathscr { H }}} } & =\left\langle J_{\mathscr{H}} x, x\right\rangle_{\mathscr{H}}=\left\langle J_{\mathscr{H}} x, x\right\rangle_{\mathscr{H}_{k}}=\left\langle J_{\mathscr{H}_{k}} x, x\right\rangle_{\mathscr{H}_{k}} \\
& =[x, x]_{\mathscr{H}_{k}} .
\end{aligned}
$$

Hence for $x \in \operatorname{span} \mathscr{H}_{k}$, we see that

$$
\begin{aligned}
\left\|C_{\infty}^{\prime} x\right\|_{\widetilde{\mathscr{K}}} & =\left\|C_{k+1} x\right\|_{\widetilde{\mathscr{K}}}=\left\|C_{k+1} x\right\|_{\mathscr{K}_{k}} \leq\left\|C_{k+1}\right\|\|x\|_{\mathscr{H}_{k}} \\
& =\left\|C_{k+1}\right\|\|x\|_{\widetilde{\mathscr{H}}}
\end{aligned}
$$

and so $C_{\infty}^{\prime}$ is a bounded operator. Since it is defined on a dense set we can extend it by continuity to a bounded operator $C_{\infty}: \widetilde{\mathscr{H}} \rightarrow \widetilde{\mathscr{K}}$. The operator $C_{\infty}$ is clearly an extension of $A_{k}$ for each $k \geq 0$ since $C_{k+1}$ is an extension of $A_{k}$ for each $k \geq 0$. To show that $C_{\infty}$ is a contraction, let $x \in \widetilde{\mathscr{H}}$. Then there exists a sequence $x_{n} \in \operatorname{span} \mathscr{H}_{k}$ such that $x_{n} \rightarrow x$. Since the inequality

$$
\left\langle C_{\infty}^{\prime} x_{n}, C_{\infty}^{\prime} x_{n}\right\rangle \leq\left\langle x_{n}, x_{n}\right\rangle
$$

holds for each $n$ we have

$$
\begin{aligned}
\left\langle C_{\infty} x, C_{\infty} x\right\rangle & =\lim _{n \rightarrow \infty}\left\langle C_{\infty}^{\prime} x_{n}, C_{\infty}^{\prime} x_{n}\right\rangle \\
& \leq \lim _{n \rightarrow \infty}\left\langle x_{n}, x_{n}\right\rangle=\langle x, x\rangle .
\end{aligned}
$$

Hence $C_{\infty}: \widetilde{\mathscr{H}} \rightarrow \widetilde{\mathscr{K}}$ is a contraction.

Define a new operator $B: \mathcal{U} \rightarrow \mathcal{Y}$ by the matrix

$$
B:=\left(\begin{array}{cc}
C_{\infty} & E \\
0 & X
\end{array}\right):\left(\begin{array}{c}
\widetilde{\mathscr{H}} \\
\mathcal{U} \ominus \widetilde{\mathscr{H}}
\end{array}\right) \longrightarrow\left(\begin{array}{c}
\widetilde{\mathscr{K}} \\
\mathscr{Y} \ominus \widetilde{\mathscr{K}}
\end{array}\right),
$$

where

$$
\left(C_{\infty} E\right)
$$

is any contractive row extension of $C_{\infty}$ and $X \in B((\mathcal{U} \ominus$ $\widetilde{\mathscr{H}}),(\mathcal{Y} \ominus \widetilde{\mathscr{K}}))$. Since $\mathcal{Y} \ominus \widetilde{\mathscr{K}}$ is a Hilbert space, Theorem 2 guarantees the existence of $X$ such that $B$ is a contraction. Note that since $\mathcal{U} \ominus \widetilde{\mathscr{H}}$ is also a Hilbert space we may set $E=0$, the zero operator, in the matrix representation of $B$ and the above result still holds.

Clearly, $B \mid \mathscr{H}_{k}=A_{k}$ and so $B$ is the required extension.

\section{Applications}

In this section we use Theorem 7 to solve a nonstationary extension problem in a Krein space setting. Let $\mathcal{U}_{k}$ and $\mathscr{Y}_{j}(j, k \in \mathbb{N})$ be Krein spaces with fixed fundamental decompositions

$$
\begin{aligned}
& \mathcal{U}_{k}=\mathcal{U}_{k}^{+} \oplus \mathcal{U}_{k}^{-}, \\
& \mathscr{Y}_{j}=\mathscr{Y}_{j}^{+} \oplus \mathcal{Y}_{j}^{-}
\end{aligned}
$$

and let

$$
f_{j, k}: \mathscr{U}_{k} \longrightarrow \mathscr{Y}_{j}
$$

be bounded operators with matrix representations

$$
f_{j, k}=\left(\begin{array}{ll}
f_{j, k}^{11} & f_{j, k}^{21} \\
f_{j, k}^{21} & f_{j, k}^{22}
\end{array}\right):\left(\begin{array}{l}
\mathcal{U}_{k}^{+} \\
\mathcal{U}_{k}^{-}
\end{array}\right) \longrightarrow\left(\begin{array}{l}
\mathscr{Y}_{j}^{+} \\
\mathcal{Y}_{j}^{-}
\end{array}\right)
$$


with $f_{j, k}^{11}=0$ for $j>k$ and $f_{j, k}^{21}=0$ for $j>0$. Define the Krein spaces $\overrightarrow{\mathscr{U}}$ and $\overrightarrow{\mathscr{y}}$ by

$$
\begin{aligned}
& \vec{u}:=\mathscr{U}_{0} \oplus \mathcal{U}_{1} \oplus \mathcal{U}_{2} \oplus \cdots, \\
& \overrightarrow{\mathcal{Y}}:=\mathscr{Y}_{0} \oplus \mathscr{Y}_{1} \oplus \mathscr{Y}_{2} \oplus \cdots
\end{aligned}
$$

and let $G: \overrightarrow{\mathscr{U}}$ to $\overrightarrow{\mathscr{Y}}$ be a bounded operator such that

$$
\left.P_{\mathscr{Y}_{j}} G\right|_{\mathscr{U}_{k}}=f_{j, k} \text {. }
$$

We use Theorem 7 to establish conditions under which such an operator $G$ is a contraction. Equality (47) implies that the operator $G$ is of the form

$$
G=\left(\begin{array}{cccccc}
\cdots & f_{4,0} & f_{3,0} & f_{2,0} & f_{1,0} & f_{0,0} \\
\cdots & f_{4,1} & f_{3,1} & f_{2,1} & f_{1,1} & f_{0,1} \\
\cdots & f_{4,2} & f_{3,2} & f_{2,2} & f_{1,2} & f_{0,2} \\
\cdots & f_{4,3} & f_{3,3} & f_{2,3} & f_{1,3} & f_{0,3} \\
\therefore & \vdots & \vdots & \vdots & \vdots & \vdots
\end{array}\right):\left(\begin{array}{c}
\vdots \\
\mathscr{u}_{4} \\
\mathcal{u}_{3} \\
\mathcal{u}_{2} \\
\mathscr{u}_{1} \\
\mathcal{u}_{0}
\end{array}\right) \longrightarrow\left(\begin{array}{c}
\mathscr{Y}_{0} \\
\mathscr{Y}_{1} \\
\mathscr{Y}_{2} \\
\mathscr{Y}_{3} \\
\mathscr{y}_{4} \\
\vdots
\end{array}\right) .
$$

Consider the decompositions

$$
\begin{aligned}
& \overrightarrow{\mathcal{U}}=\cdots \oplus \mathcal{U}_{1}^{-} \oplus \mathcal{U}_{0}^{-} \oplus \mathcal{U}_{0}^{+} \oplus \mathcal{U}_{1}^{+} \oplus \cdots, \\
& \vec{y}=\cdots \oplus \mathcal{Y}_{1}^{-} \oplus \mathcal{Y}_{0}^{-} \oplus \mathcal{Y}_{0}^{+} \oplus \mathscr{Y}_{1}^{+} \oplus \cdots .
\end{aligned}
$$

With the above decompositions, the operator $G$ takes the form

$$
\begin{aligned}
& G=\left(\begin{array}{ccccc|ccccc}
\ddots & \vdots & \vdots & \vdots & \vdots & \vdots & \vdots & \vdots & \vdots & \ddots \\
\cdots & f_{2,3}^{11} & f_{2,2}^{11} & 0 & 0 & 0 & 0 & 0 & 0 & \cdots \\
\cdots & f_{1,3}^{11} & f_{1,2}^{11} & f_{1,1}^{11} & 0 & 0 & 0 & 0 & 0 & \cdots \\
\cdots & f_{0,3}^{11} & f_{0,2}^{11} & f_{0,1}^{11} & f_{0,0}^{11} & f_{0,0}^{12} & f_{0,1}^{12} & f_{0,2}^{12} & f_{0,3}^{12} & \cdots \\
\hline \cdots & f_{0,3}^{21} & f_{0,2}^{21} & f_{0,1}^{21} & f_{0,0}^{21} & f_{0,0}^{22} & f_{0,1}^{22} & f_{0,2}^{22} & f_{0,3}^{22} & \cdots \\
\cdots & f_{1,3}^{11} & f_{1,2}^{11} & f_{1,1}^{11} & f_{1,0}^{11} & f_{1,0}^{22} & f_{1,1}^{22} & f_{1,2}^{22} & f_{1,3}^{22} & \cdots \\
\cdots & \vdots & \vdots & \vdots & \vdots & \vdots & \vdots & \vdots & \vdots & \vdots
\end{array}\right): \\
& \left(\begin{array}{c}
\vdots \\
\mathcal{U}_{2}^{+} \\
\mathcal{U}_{1}^{+} \\
\mathcal{U}_{0}^{+} \\
\mathcal{U}_{0}^{-} \\
\mathcal{U}_{1}^{-} \\
\mathcal{U}_{2}^{-} \\
\vdots
\end{array}\right) \longrightarrow\left(\begin{array}{c}
\vdots \\
y_{2}^{+} \\
\mathscr{Y}_{1}^{+} \\
\frac{y_{0}^{+}}{\mathscr{Y}_{0}^{-}} \\
\mathscr{Y}_{1}^{-} \\
\mathscr{Y}_{2}^{-} \\
\vdots
\end{array}\right) .
\end{aligned}
$$

For each $k \in \mathbb{N}$, let $A_{k}$ denote the operator

$A_{k}$

$$
\begin{aligned}
& =\left(\begin{array}{ccccc|ccccc}
f_{k, k}^{11} & 0 & \cdots & 0 & 0 & 0 & 0 & 0 & 0 & \cdots \\
\vdots & \ddots & \vdots & \vdots & \vdots & \vdots & \vdots & \vdots & \vdots & \vdots \\
f_{2, k}^{11} & \cdots & f_{2,2}^{11} & 0 & 0 & 0 & 0 & 0 & 0 & \cdots \\
f_{1, k}^{11} & \cdots & f_{1,2}^{11} & f_{1,1}^{11} & 0 & 0 & 0 & 0 & 0 & \cdots \\
f_{0, k}^{11} & \cdots & f_{0,2}^{11} & f_{0,1}^{11} & f_{0,0}^{11} & f_{0,0}^{12} & f_{0,1}^{12} & f_{0,2}^{12} & f_{0,3}^{12} & \cdots \\
\hline f_{0, k}^{21} & \cdots & f_{0,2}^{21} & f_{0,1}^{21} & f_{0,0}^{21} & f_{0,0}^{22} & f_{0,1}^{22} & f_{0,2}^{22} & f_{0,3}^{22} & \cdots \\
f_{1, k}^{21} & \cdots & f_{1,2}^{21} & f_{1,1}^{21} & f_{1,0}^{21} & f_{1,0}^{22} & f_{1,1}^{22} & f_{1,2}^{22} & f_{1,3}^{22} & \cdots \\
\vdots & \vdots & \vdots & \vdots & \vdots & \vdots & \vdots & \vdots & \vdots & \vdots
\end{array}\right) \\
& \left(\begin{array}{c}
u_{k} \\
\vdots \\
\mathcal{U}_{2}^{+} \\
\mathcal{U}_{1}^{+} \\
\mathcal{U}_{0}^{+} \\
\mathcal{U}_{0}^{-} \\
\mathcal{U}_{1}^{-} \\
\mathscr{U}_{2}^{-} \\
\vdots
\end{array}\right) \longrightarrow\left(\begin{array}{c}
\mathscr{Y}_{k} \\
\vdots \\
\mathscr{Y}_{2}^{+} \\
\mathscr{Y}_{1}^{+} \\
\mathscr{Y}_{0}^{+} \\
\mathscr{Y}_{0}^{-} \\
\mathscr{Y}_{1}^{-} \\
\mathscr{Y}_{2}^{-} \\
\vdots
\end{array}\right) .
\end{aligned}
$$

With these notations, we are in a position to state the following theorem.

Theorem 8. Let $\mathscr{U}_{k}$ and $\mathscr{Y}_{j}$ be Krein spaces with decompositions (43) with operators $f_{j, k}: \mathscr{U}_{k} \rightarrow \mathscr{Y}_{j}$ in (44). Then there exists a contraction $G: \overrightarrow{\mathcal{U}} \rightarrow \vec{y}$ satisfying (47) if and only if, for each $k \in \mathbb{N}$, the operator $A_{k}$ in (51) defines a contraction whose norm has an upper bound independent of $k$. 
Proof. Assume that a contraction $G: \overrightarrow{\mathscr{U}} \rightarrow \vec{y}$ satisfying (47) exists and consider the operator matrices (50) and (51). We see that, for $k \in \mathbb{N}$,

$$
A_{k}=\left.P_{\mathscr{K}_{k}} G\right|_{\mathscr{H}_{k}}
$$

where

$$
\begin{gathered}
\mathscr{H}_{k}=\left(\begin{array}{c}
\mathscr{U}_{k} \\
\vdots \\
\mathcal{U}_{1}^{+} \\
\mathcal{U}_{0}^{+} \\
\mathcal{U}_{0}^{-} \\
\mathcal{U}_{1}^{-} \\
\vdots \\
\mathscr{K}_{k}= \\
\mathscr{Y}_{k} \\
\vdots \\
\mathscr{Y}_{1}^{+} \\
\mathscr{Y}_{0}^{+} \\
\mathscr{Y}_{0}^{-} \\
\mathscr{Y}_{1}^{-} \\
\vdots
\end{array}\right),
\end{gathered}
$$

Since $\overrightarrow{\mathscr{Y}} \ominus \mathscr{K}_{k}$ is a Hilbert space, we see that $\left\|P_{\mathscr{K}_{k}}\right\| \leq 1$ and so

$$
\left\|A_{k}\right\| \leq\left\|P_{\mathscr{K}_{k}}\right\|\|G\| \leq\|G\| .
$$

This shows that the operators $A_{k}$ are uniformly bounded. Since $G$ is a contraction and $\overrightarrow{\mathscr{Y}} \ominus \mathscr{K}_{k}$ is a Hilbert space, (52) implies that $A_{k}$ is a contraction for each $k \in \mathbb{N}$.

To prove the reverse implication, we assume that for each $k \in \mathbb{N}$ the operator $A_{k}: \mathscr{H}_{k} \rightarrow \mathscr{K}_{k}$ is a contraction of norm at most $\gamma$ where $0<\gamma<\infty$. Since span $\mathscr{H}_{k}=\overrightarrow{\mathscr{U}}$ and span $\mathscr{K}_{k}=\overrightarrow{\mathscr{Y}}$, it follows that $\overline{\operatorname{span}} \mathscr{H}_{k}$ and $\overline{\operatorname{span}} \mathscr{K}_{k}$ are regular subspaces. By construction, the subspaces $\mathscr{H}_{k}$ and $\mathscr{K}_{k}$ satisfy the conditions of Corollary 6 and hence do satisfy all the conditions specified in Theorem 7. Since, for each $k \in \mathbb{N}$, the operators

$$
A_{k}: \mathscr{H}_{k} \longrightarrow \mathscr{K}_{k}
$$

are defined by (51) and are assumed to be uniformly bounded, condition (31) in Theorem 7 is also fulfilled (it can be easily seen that the first condition in (31) is satisfied by the operators $A_{k}$ ). Since (31) is satisfied for all $k \in \mathbb{N}$, it follows that there exists a contraction $B$ satisfying (30) and therefore must be of the form (50). This concludes the proof.

\section{Conflicts of Interest}

The author declares that there are no conflicts of interest regarding the publication of this paper.

\section{References}

[1] G. Arsene, T. Constantinescu, and A. Gheondea, "Lifting of operators and prescribed numbers of negative squares," Michigan Mathematical Journal, vol. 34, no. 2, pp. 201-216, 1987.

[2] M. A. Dritschel and J. Rovnyak, "Extension theorems for contraction operators on Krein spaces," Operator Theory: Advances and Applications, vol. 47, pp. 221-305, 1990.

[3] M. A. Dritschel, "A lifting theorem for bicontractions on Kreǐn spaces," Journal of Functional Analysis, vol. 89, no. 1, pp. 61-89, 1990.

[4] A. Gheondea, "Canonical forms of unbouded unitary operators in Krein operators," Publications of the Research Institute for Mathematical Sciences, vol. 24, no. 2, pp. 205-224, 1988.

[5] A. Gheondea, "On the structure of linear contractions with respect to fundamental decompositions," Operator Theory: Advances and Applications, vol. 48, pp. 275-295, 1990.

[6] C. Foias, A. E. Frazho, I. Gohberg, and M. . Kaashoek, "Metric constrained interpolation, commutant lifting and systems," Operator Theory: Advances and Applications, vol. 100, 1998.

[7] C. Foias, A. E. Frazho, I. Gohberg, and M. . Kaashoek, "Parameterization of all solutions of the three chains completion problem," Integral Equations and Operator Theory, vol. 29, no. 4, pp. 455-490, 1997.

[8] C. Foias, A. E. Frazho, I. Gohberg, and M. . Kaashoek, "A time-variant version of the commutant lifting theorem and nonstationary interpolation problems," Integral Equations and Operator Theory, vol. 28, no. 2, pp. 158-190, 1997.

[9] T. Ando, Linear Operators on Krein Spaces, Hokkaido University, Research Institute of Applied Electricity, Division of Applied Mathematics, Sapporo, Japan, 1979.

[10] T. J. Azizov and I. S. Iohvidov, "Linear operators in spaces with an indefinite metric," Itogi Nauki i Techniki, pp. 113-205, 272, 1979.

[11] J. Bognar, Indefinite Inner Product Spaces, Springer-Verlag, New York, NY, USA, 1974.

[12] I. S. Iohvidov, M. G. Krein, and H. Langer, Introduction to The Spectral Theory of Operators in Spaces with An Indefinite Metric, vol. 9 of Mathematical Research, Akademie-Verlag, Berlin, Germany, 1982.

[13] G. Arsene and A. Gheondea, "Completing matrix contractions," The Journal of Operator Theory, vol. 7, no. 1, pp. 179-189, 1982.

[14] T. Constantinescu and A. Gheondea, "Minimal signature in lifting of operators I," The Journal of Operator Theory, vol. 22, pp. $345-367,1989$. 


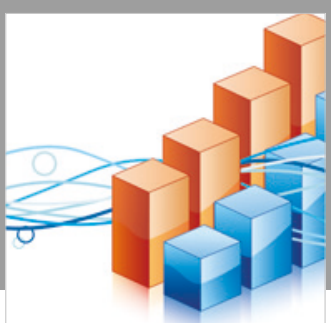

Advances in

Operations Research

\section{-n-m}
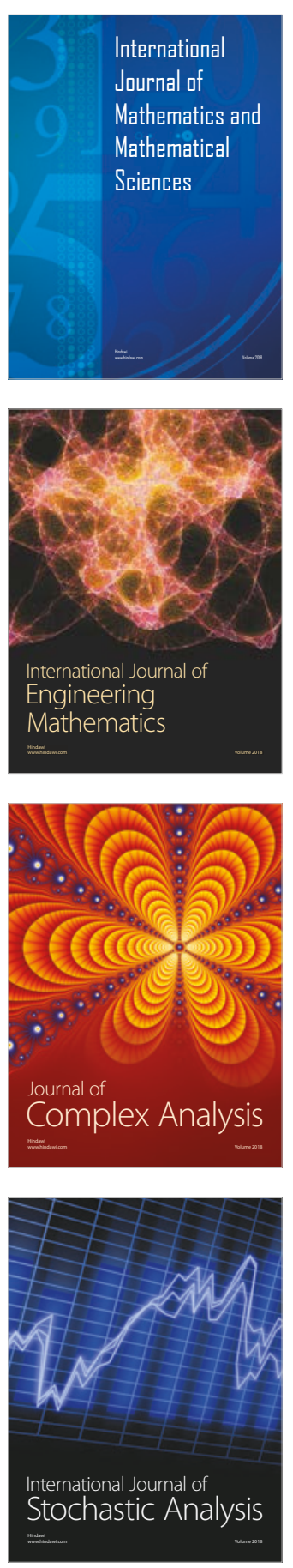
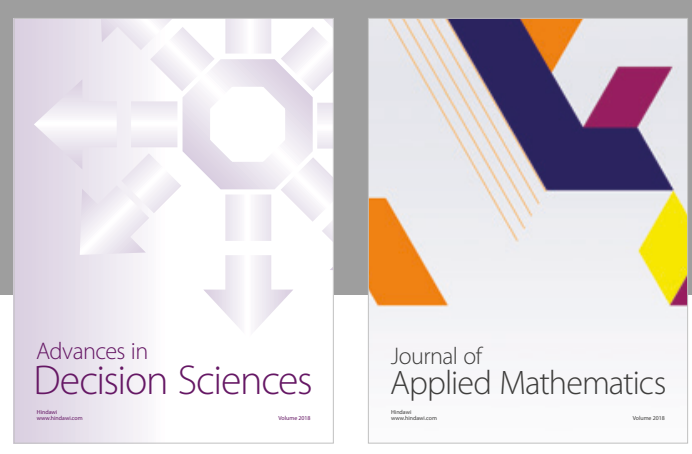

Journal of

Applied Mathematics
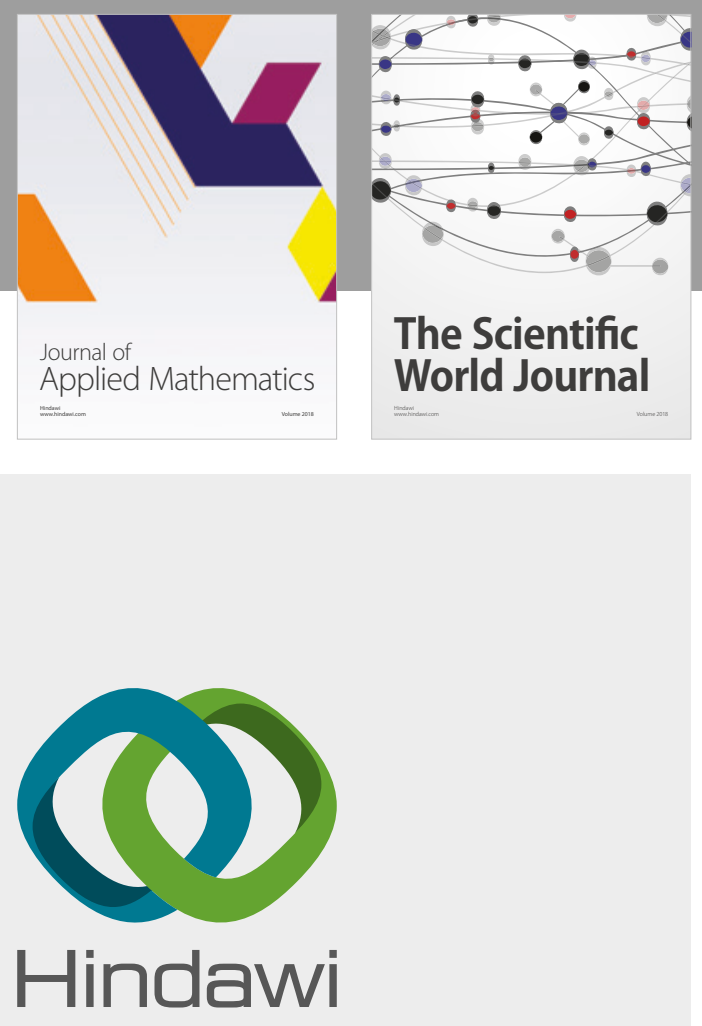

Submit your manuscripts at

www.hindawi.com

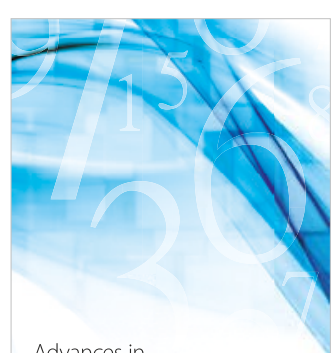

Advances in
Numerical Analysis
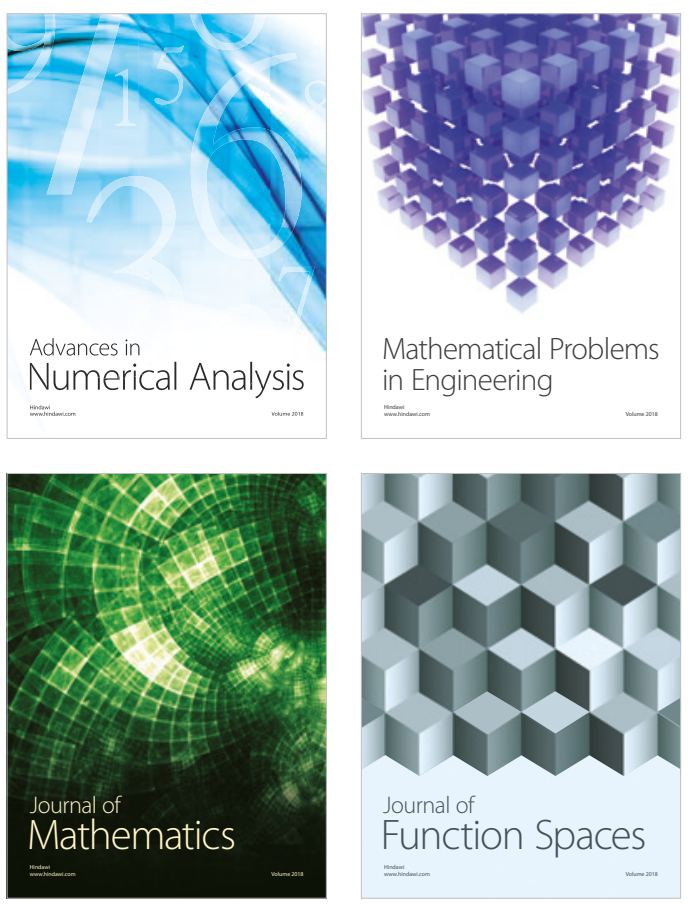

Mathematical Problems in Engineering

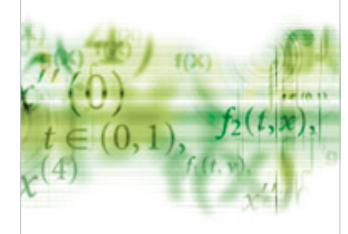

International Journal of

Differential Equations

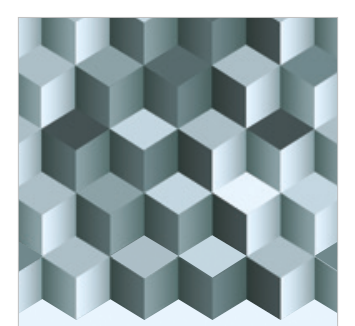

Journal of

Function Spaces

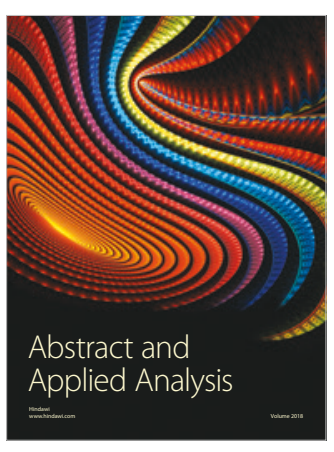

The Scientific

World Journal

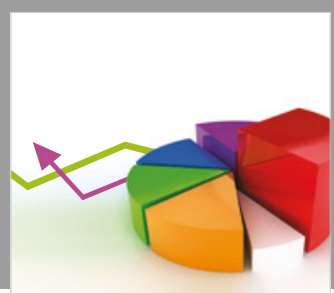

Journal of

Probability and Statistics
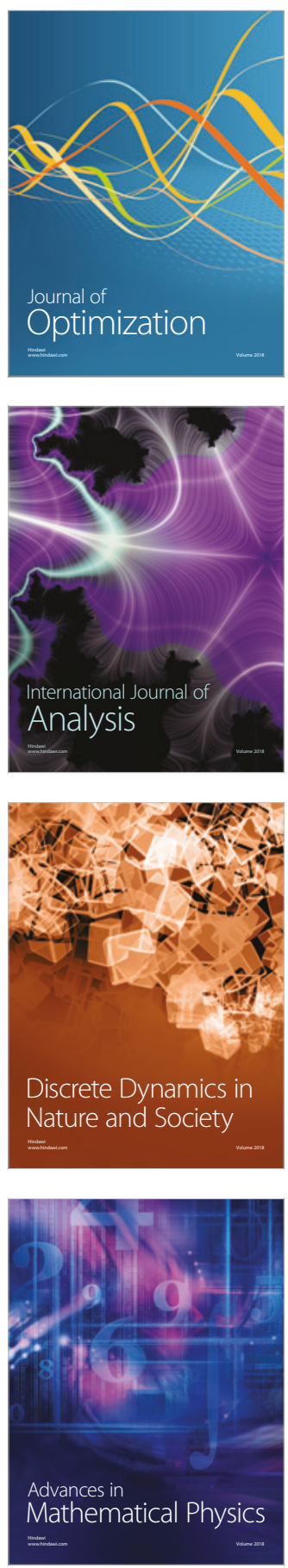